\title{
An alternative approach for the determination of mean free paths of electron scattering in liquid water based on experimental data
}

\author{
Axel Schild, ${ }^{*, \dagger}$ Michael Peper, ${ }^{\dagger}$ Conaill Perry, ${ }^{\dagger}$ Dominik Rattenbacher, ${ }^{\dagger}, \ddagger$ and \\ Hans Jakob Wörner ${ }^{\dagger}$ \\ $\dagger$ ETH Zürich, Laboratorium für Physikalische Chemie, 8093 Zürich, Switzerland \\ $\ddagger$ Max Planck Institute for the Science of Light, Staudtstr. 2, 91058 Erlangen, Germany \\ E-mail: axel.schild@phys.chem.ethz.ch
}

\begin{abstract}
The mean free paths of low-energy electrons in liquid water are of fundamental importance for modelling radiation damage and many related physico-chemical processes. Neither theoretical predictions nor experimental estimations have so far converged to yield reliable values for these parameters. We therefore introduce a new approach to determine the elastic and inelastic mean free paths (EMFP, IMFP) based on experimental data. We report extensive abinitio calculations of electron quantum scattering with water clusters, which are brought to convergence with respect to the cluster size. This provides a first-principles approach to condensed-phase scattering that includes both multiple-scattering and condensation effects. The obtained differential cross sections are used in a detailed Monte-Carlo simulation to extract EMFP and IMFP from two recent liquidmicrojet experiments that determined the effective attenuation length (EAL) and the photoelectron angular distribution (PAD) following oxygen 1s-ionization of liquid water. For electron kinetic energies from $10 \mathrm{eV}$ to $300 \mathrm{eV}$, we find that the IMFP is noticeably larger than the EAL. The EMFP is longer than that of gasphase water and the IMFP is longer compared to the latest theoretical estimations, but both the EMFP and IMFP are much shorter than
\end{abstract}

suggested by experimental results for amorphous ice. The Python module developed for the analysis is available at https://gitlab. com/axelschild/CLstunfti and can be used to further refine our results when new experimental data become available.

\section{Article}

Knowledge of the scattering properties of electrons in liquid water is vital for understanding and for modeling many physical processes, for example the effect of radiation damage in living tissue. ${ }^{[13}$ To accurately model the interaction of electrons with water molecules, e.g. with Monte-Carlo simulations, $\frac{415}{15}$ the differential scattering cross sections (DCS) as well as the total cross sections for many possible types of interactions are needed. Those quantities are experimentally accessible for gas-phase water, ${ }^{6}[9]$ but for water in the liquid phase they are difficult to obtain.

Instead of the cross sections, the elastic mean free path (EMFP) and the inelastic mean free path (IMFP) for a given electron kinetic energy (eKE) can be used as a measure for the probability to scatter. The IMFP is a particularly relevant parameter because it represents the effective path length that an electron can travel in liquid water before it scatters inelastically, thereby losing some of its energy and 
thus changing the probability of damaging solvated molecules. Hence, there has been much interest in determining the IMFP theoretically as well as experimentally: On the one hand, there have been a number of theoretical estimations of the IMFP. $10 \sqrt{22}$ Especially for eKE below $100 \mathrm{eV}$, the often utilized models, which are based on the dielectric function, have limitations that need to be overcome. These include, e.g., the neglect of electron-exchange effects and an overestimation of the needed energyloss function in the presence of an energy gap in the excitation spectrum (see Emfietzoglou et al. $\frac{18}{18}$ and Nguyen-Truong ${ }^{22}$ for details). On the other hand, the mean free path is a quantity that eludes direct measurements. What has been measured directly, however, are the effective attenuation lengths (EAL) of electrons in liquid water 23 and the photoelectron angular distributions (PAD) of electrons generated through core-level ionization inside liquid water. ${ }^{[26}$ While the EAL provides a lower bound for the IMFP, the measured PAD of liquid water was used to estimate the IMFP directly with a simplified model. It was found that for an eKE below $\approx 100 \mathrm{eV}$, the IMFP appears to be considerably shorter and flatter than previously assumed. ${ }^{26}$ A recent improvement of the theoretical algorithms used to determine the IMFP within the dielectric formalism yielded results that qualitatively agree with the experimental results. 22

There have also been attempts to determine scattering cross sections from photoelectron imaging of water droplets ${ }^{27}$ with the surprising finding that the IMFP and scattering cross sections ${ }^{28}$ were identical to those measured for amorphous ice ${ }^{29}$ within the quoted accuracies. In a subsequent work,, 30 attempts were made to explain angle-resolved photoelectronimaging results for water clusters with the cross sections, but they had to be rescaled to qualitatively reproduce the experimental results. Discussions in the literature ${ }^{19131}$ show that it is thus appropriate to conclude that there is no consensus regarding the EMFP and IMFP values for electron scattering in liquid water, neither from theoretical nor from experimental approaches. This situation motivates the present work.

In the following, we present an alternative approach to determine the EMFP and IMFP of electron scattering in liquid water based on experimental data. Our approach relies on extensive ab-initio quantum-scattering calculations of electrons with water clusters of variable size to achieve a first-principles description of electron scattering in liquid water. The approach includes, at a quantum-mechanical level, the key characteristics that distinguish electron scattering in the gas and condensed phases. These differences originate from (i) multiplescattering and interference effects originating from scattering at neighboring molecules in the condensed phase and (ii) the modification of the electronic structure of the water molecules through condensation. Verifying the convergence of our scattering calculations with respect to cluster size ensures that the relevant effects have been taken into account.

We then use Monte-Carlo electron-trajectory calculations to connect these ab-initio results with experimental observables. To uniquely determine EMFP and IMFP values which are consistent with experimental observations, we need two independent measurements. For this purpose, we chose the measurements of the EAL by Suzuki et al. ${ }^{25}$ and of the PAD by Thürmer et al. ${ }^{26}$, both realized by ionizing the oxygen 1s-orbital of liquid water in microjets. We use classical-trajectory Monte-Carlo simulations to describe the electron transport. Such calculations are expected to be valid for kinetic energies above ca. $10 \mathrm{eV}$, where quantuminterference effects have been shown to become negligible. ${ }^{32}$ In our approach, the only free parameters are the EMFP and the IMFP, while all other input parameters are determined from $a b$ initio calculations or from the measurements. The assumptions made in the Monte-Carlo simulations are described in the supplementary material.

The EMFP and IMFP are defined by assuming that for both elastic and inelastic scattering, the probability of not having scattered until a 
distance $r$ is

$$
P(r)=\frac{1}{r_{\mathrm{MFP}}} e^{-r / r_{\mathrm{MFP}}} .
$$

Then, $\int_{0}^{\infty} r P(r) d r=r_{\mathrm{MFP}}$ is the corresponding mean free path.

The EAL is a distance parameter that describes the exponential decay of the number of electrons $S(z)$ detected outside the liquid,

$$
S(z) \propto \exp \left(-z / r_{\mathrm{EAL}}\right),
$$

as a function of the distance $z$ from their point of creation to the surface. It is measured for an eKE corresponding to the kinetic energy after ionization. Since the depth of the conduction band (the electron affinity) of liquid water is well below $1 \mathrm{eV}, 31 / 33$ we neglect it due to the comparably high $\mathrm{eKE} \geq 10 \mathrm{eV}$ that we consider. The EAL is related to the IMFP, because inelastic scattering is responsible for the loss of signal $S(z)$ with starting depth $z$ of the electron, and the EAL is a lower bound for the IMFP.

For an isotropic sample ionized with linearly polarized light, the PAD relative to the direction of polarization is given by

$$
\operatorname{PAD}(\theta) \propto 1+\beta P_{2}(\cos \theta)
$$

where $\theta$ is the polar angle, $P_{2}$ is the Legendre polynomial of second order, and $\beta$ is the asymmetry parameter. The PAD of photoemission from a dense medium is closely related to the mean number of elastic collisions before inelastic scattering occurs,

$$
\left\langle N_{\text {ela }}\right\rangle=\frac{r_{\mathrm{IMFP}}}{r_{\mathrm{EMFP}}}
$$

because each elastic collision changes the angular distribution of the electrons at the observed eKE.

The DCS for electronically, vibrationally, and rotationally elastic $\left(J=0 \rightarrow J^{\prime}=0\right)$ scattering are computed with the program ePolyScat ${ }^{37 / 38}$ for water clusters of different sizes and shapes with nuclear configurations taken from Temelso et al. $\stackrel{39}{ }$, and also for some structures based on the modeling of experimental data for the first solvation shell of liquid water. ${ }^{40}$ ePolyScat solves the variational Schwinger equation using single-center expansions, and we use molecularorbital data from Hartree-Fock calculations with a cc-pVTZ basis set obtained with the program Gaussian $\underline{41}$ as input.

The most important result from these calculations is that the DCS rapidly converge with the number of water molecules in the cluster (see Fig. S1 of the supplemental material). With increasing kinetic energy, the DCS are found to converge more rapidly as a function of cluster size. These observations can be intuitively explained by relating the de-Broglie wavelength $\lambda_{\mathrm{dB}}$ of the scattering particle to the extension of the target. Whereas the molecularlevel description of a $10 \mathrm{eV}$ electron $\left(\lambda_{\mathrm{dB}} \approx\right.$ $3.9 \AA)$ collision requires a cluster of $6-7$ water molecules to reach convergence, a $50 \mathrm{eV}$ electron $\left(\lambda_{\mathrm{dB}} \approx 1.7 \AA\right)$ collision is well described by a much smaller cluster. The observed rapid convergence of the DCS suggests that such cluster calculations can be a good approximation for the DCS of bulk liquids. ${ }^{42}$ In the present work, we used the DCS of the largest considered cluster, $\left(\mathrm{H}_{2} \mathrm{O}\right)_{7}$, to describe the DCS of bulk water.

The Monte-Carlo trajectory calculations were realized with the Python module CLstunfti, which is available at https://gitlab.com/ axelschild/CLstunfti. Here, we only give a brief overview of the computational model: Electrons at a given eKE are represented by classical trajectories and a set of EMFP and IMFP are chosen for the simulation. To simulate ionization, an ionization depth $z$ is selected and initial directions for the trajectories are sampled from the experimental gas-phase PAD. To account for inelastic scattering, each trajectory is given a random maximum path length to travel according to the distribution (1) and using $r_{\mathrm{MFP}}=r_{\mathrm{IMFP}}$. It is assumed that inelastic scattering happens at this distance and that if this happens, the electron is no longer detected within the relevant eKE range. This separation of scattering into elastic and inelastic events is based on the experimental observation of well separated groups of primary and secondary electrons. Only the primary electrons, which form a well-defined Gaussian dis- 
tribution of $\sim 1.5 \mathrm{eV}$ width are detected in the simulated photoelectron experiments. The secondary electrons, which have experienced one or more electronically inelastic scattering events corresponding to an energy loss of $7 \mathrm{eV}$ or more, are not considered. To propagate the trajectories after the ionization event, a travel distance is selected randomly for each trajectory according to the distribution (1) with $r_{\mathrm{MFP}}=r_{\mathrm{EMFP}}$. If the trajectory ends outside the liquid (the liquid surface is located at $z=0$, i.e., it is flat) or if its path is longer than the maximum path length for the trajectory (i.e., when inelastic scattering would happen), it is counted to the measured signal or it is discarded, respectively. If this is not the case, elastic scattering happens, the position of the trajectory is updated and a new direction is chosen according to the DCS. This last step is repeated until all trajectories have either reached the surface (contributing to the measured signal) or have been discarded due to their path being longer than the pre-selected maximum path.

To simulate the EAL measurement, the ionizing beam is assumed to have a polarization vector that is directed to the surface and the number of escaped electrons is counted depending on the chosen starting depth $z$ of the trajectories. For the simulation of the PAD of the liquid phase, random starting depths are chosen. As done in the experiment, the direction of the polarization vector of the ionizing beam is varied. In both experiments, the escaped electrons are counted if they exit with a small polar angle. Ideally, the detection angle should be very small. In the simulations we chose it to be $1^{\circ}$, but the results discussed in the following are not very sensitive to this angle as long as it is chosen to be small enough, and test calculations with opening angles of $15^{\circ}$ (which are approximately the experimental opening angles) yield similar numerical results compared to the ones presented below. We also use importance sampling with an exponential distribution for the initial starting depths of the trajectories to make the simulation more efficient, as trajectories starting deep inside the liquid have a low probability of reaching the surface due to inelastic scattering.
We start the discussion of the results with those observations that we expect to have a general character and could thus be useful in interpreting other experimental results. To the best of our knowledge, these dependencies have not been reported before. Figure 1 illustrates the dependence of the experimental observables EAL and $\beta$ on the EMFP and the average number of elastic scatterings $\left\langle N_{\text {ela }}\right\rangle$ for an eKE of $20 \mathrm{eV}$. We recall that $\left\langle N_{\text {ela }}\right\rangle$ encodes the dependence on the IMFP via (4). We find that (i) for fixed EMFP the EAL is increasing as $\sqrt{\left\langle N_{\text {ela }}\right\rangle}$, (ii) for fixed EMFP the parameter $\beta$ is decreasing as $1 /\left\langle N_{\text {ela }}\right\rangle$ within the investigated range (top and bottom-left panels of Fig. 1), (iii) for fixed $\left\langle N_{\text {ela }}\right\rangle$ the EAL is proportional to the EMFP, and (iv) for fixed $\left\langle N_{\text {ela }}\right\rangle$ the parameter $\beta$ is independent of the EMFP (top and bottom-right panels of Fig. 11). The reasons for these dependencies are the following: The (in)dependencies (iii) and (iv) on the EMFP can be explained by a scaling argument: For fixed $\left\langle N_{\text {ela }}\right\rangle$, it follows from (4) that trajectories from a simulation with $r_{\mathrm{EMFP}}=r_{2}$ are equivalent to those for $r_{\mathrm{EMFP}}=r_{1}$ if scaled by the factor $r_{2} / r_{1}$. The scaling leaves the final PAD invariant and changes the depth distribution from which the measured trajectories originate linearly with the EMFP. The linear dependence (i) of the EAL on $\sqrt{\left\langle N_{\text {ela }}\right\rangle}$ is due to scattering of trajectories into other directions than the forward direction - the EAL is for the trajectories like the root mean square translation distance of a random walk, which also scales like $\sqrt{n}$ where $n$ is the number of steps. ${ }^{43}$ All dependencies are monotonic, hence a set of values for the EAL and $\beta$ corresponds to a unique set of values for the EMFP and IMFP. These dependencies also illustrate a general protocol for analyzing the experimental results. The measurement of $\beta$ uniquely defines $\left\langle N_{\text {ela }}\right\rangle$, i.e. the ratio of IMFP to EMFP. Knowing $\left\langle N_{\text {ela }}\right\rangle$, the measurement of the EAL uniquely defines the EMFP, which in turn defines the IMFP. As a consequence, an overestimation of the EAL would result in EMFP and IMFP values that are both too long by the same factor. Errors in the measurement of $\beta$ would affect the ratio of IMFP to EMFP.

We now turn to the discussion of the mean 

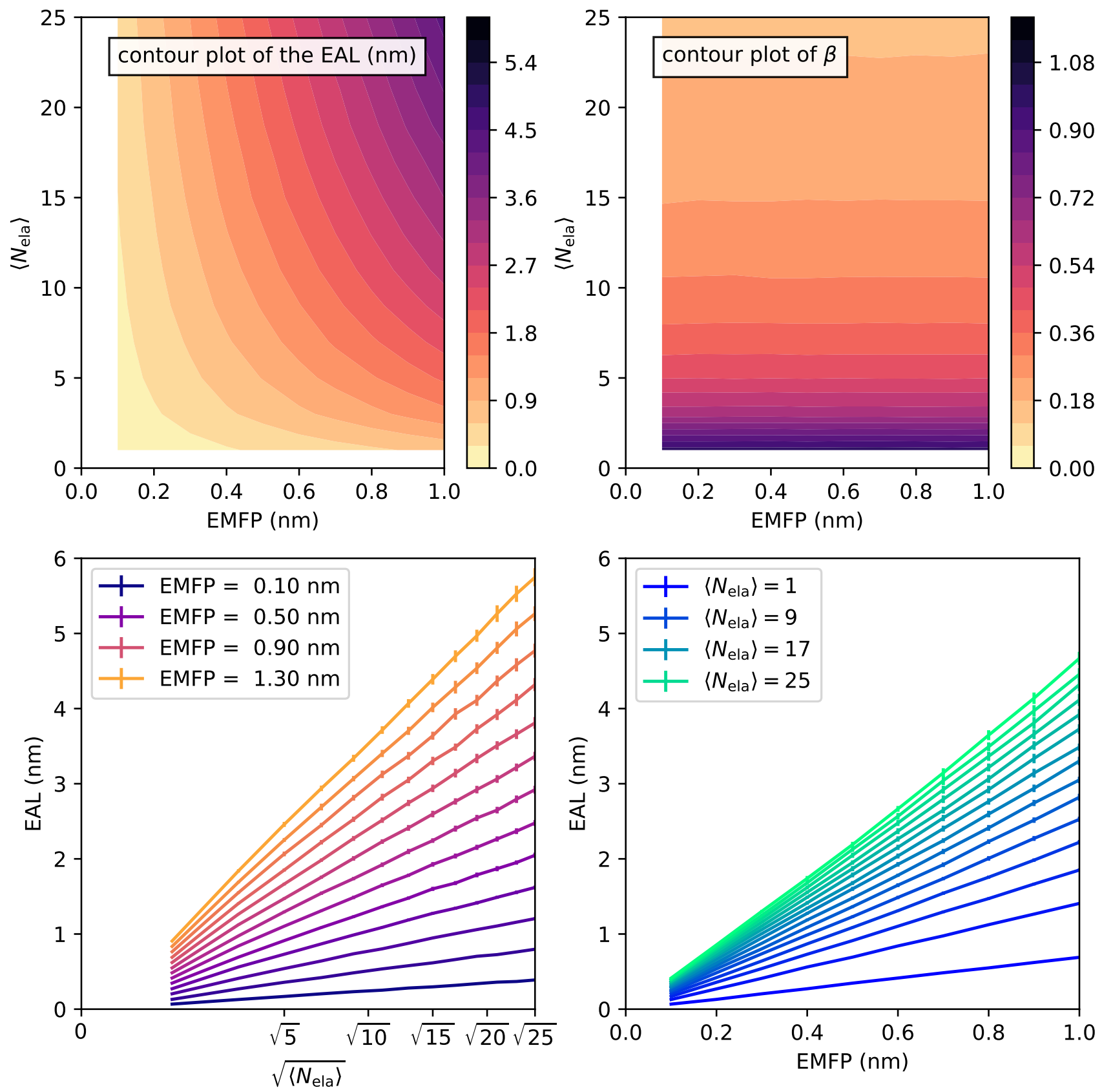

Figure 1: Top: Contour plots of the effective attenuation length EAL (left) and the $\beta$-parameter describing the photoelectron angular distribution of the liquid (right) depending on the value of the elastic mean free path (EMFP) and the average number of elastic collisions $\left\langle N_{\text {ela }}\right\rangle$. Bottom: Line plots of the EAL depending on $\sqrt{\left\langle N_{\text {ela }}\right\rangle}$ for fixed EMFP (left) and depending on the EMFP for fixed $\left\langle N_{\text {ela }}\right\rangle$ (right). Small vertical lines indicate the standard deviation of the fit to (2). The plots show results for an electron kinetic energy of $20 \mathrm{eV}$ and the differential scattering cross sections obtained with ePolyScat for $\left(\mathrm{H}_{2} \mathrm{O}\right)_{7}$ clusters. 

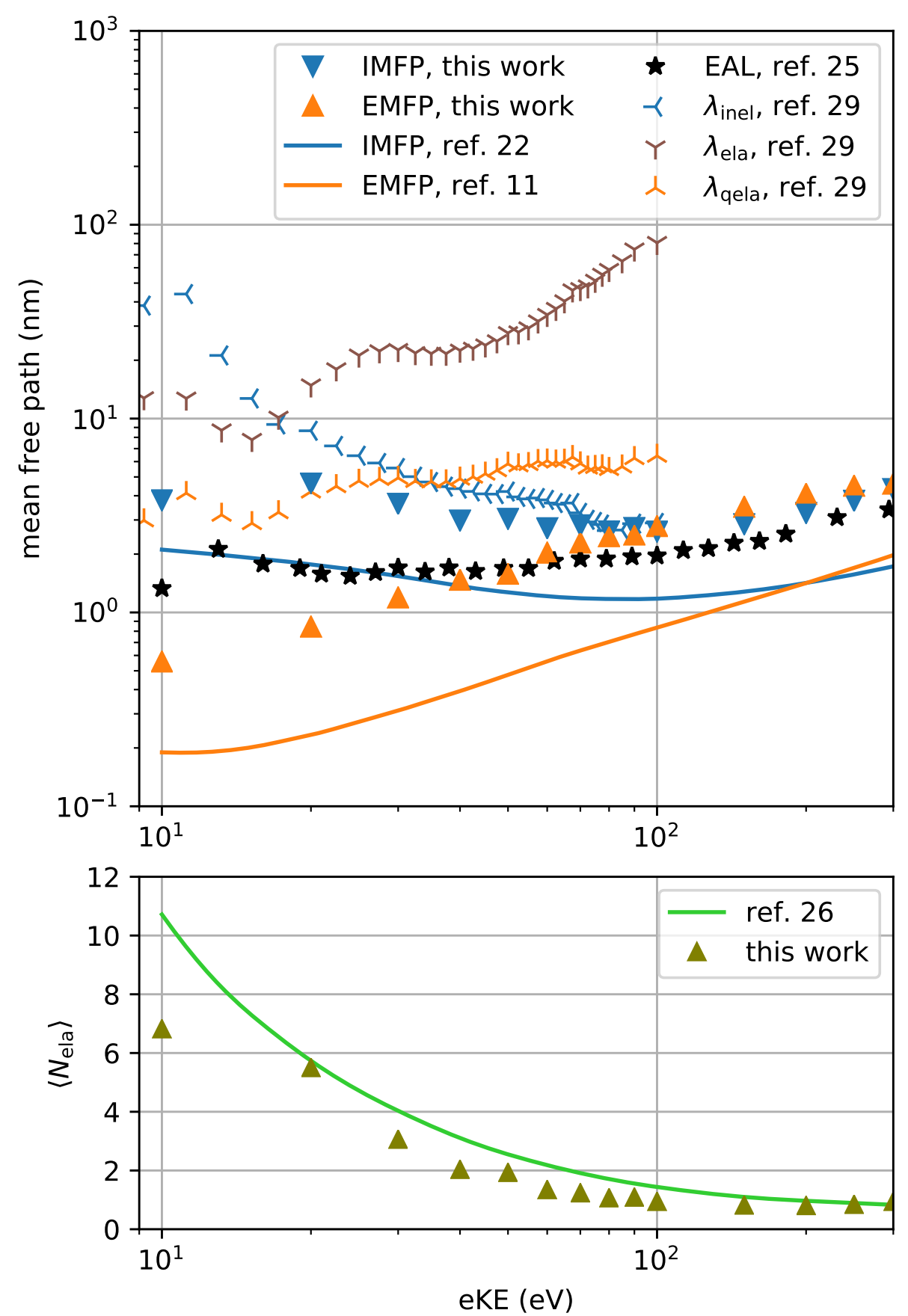

Figure 2: Top: Elastic (EMFP) and inelastic (IMFP) mean free paths from our simulation. For comparison, the measured elastic mean free paths (EAL, extracted from Suzuki et al. ${ }^{(25)}$ ) as well as the EMFP from Tomita et al.111 (corresponding to the integrated scattering cross sections for gasphase water from Märk et al. $\left.{ }^{44}\right)$, the IMFP from Nguyen-Truong ${ }^{22}$, and mean free paths obtained from Michaud et al. ${ }^{29}$ for amorphous ice are shown. The elastic $\left(\lambda_{\text {ela }}\right)$, quasi-elastic $\left(\lambda_{\text {qela }}\right)$, and inelastic $\left(\lambda_{\text {inel }}\right)$ mean free paths for Michaud et al. ${ }^{29}$ are obtained from the cross sections of the first column of Table 2, the sum of the cross sections for all columns of Table 2 and of all but "Others" in Table 3, and the cross sections "Others" in Table 3 of that reference, respectively. Bottom: Average number of elastic collisions $\left\langle N_{\text {ela }}\right\rangle$. The values from Thürmer et al. ${ }^{26}$ are shown for comparison. 
free paths obtained from the analysis of the experimental results. The top panel of Figure 2 shows the dependencies of the retrieved EMFP and IMFP on the eKE. For comparison, the experimentally determined EAL used in the simulations is shown, as well as literature values for the EMFP corresponding to the total elastic scattering cross section for gas-phase water, 11144 and the most recent theoretical estimation of the IMFP for liquid water. ${ }^{22}$ Figure 2 also contains the mean free paths for purely elastic, quasi-elastic (i.e. including vibrationally inelastic) and electronically inelastic scattering determined by Michaud et al. $\frac{29}{}$ from experiments on amorphous ice. We note that the cross sections reported by Luckhaus et al. ${ }^{28}$ are identical to those of Michaud et al. ${ }^{29}$ for all energies (from $1.7 \mathrm{eV}$ upwards) reported in the latter. A comparison to the many other estimations of the IMFP can e.g. be found in Nguyen-Truong 22 .

The EMFP determined in our work (cyan triangles) is larger by a factor of 2-3 compared to previous estimates based on gas-phase data (cyan line). It is smaller by a factor of $2-4$ than the quasi-elastic MFP of Michaud et al. ${ }^{29}$ (cyan tripods). We note that the purely elastic EMFP

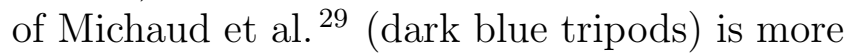
than one order of magnitude longer than our EMFP and up to 2 orders of magnitude longer than the previous estimates.

The IMFP determined in our work (magenta triangles) is found to be longer than the latest theoretical estimate ${ }^{22}$ (magenta line) by a nearly constant factor of $\sim 2$. Our IMFP is smaller than the electronic IMFP determined by Michaud et al. ${ }^{29}$ by up to one order of magnitude at $10 \mathrm{eV}$, but seems to merge with the latter around $80 \mathrm{eV}$. Our IMFP is larger than the experimental EAL by a factor of $\sim 3$ for an eKE of $10 \mathrm{eV}$, getting closer to the EAL for higher eKE, but always remaining larger than the EAL, as required. In our simulations, we find that the EAL becomes equal to the IMFP only if we set the EMFP to be very long such that no elastic scattering happens at all.

For eKE $\geq 60 \mathrm{eV}$, both the IMFP and EMFP are larger than the EAL and comparable in magnitude. The EMFP eventually becomes somewhat larger than the IMFP for eKE $\geq$
$100 \mathrm{eV}$, which is lower than the crossing point of the previous estimates around $200 \mathrm{eV}$. This does however not mean that elastic scattering becomes unimportant in the photoelectron experiments. A significant influence of elastic scattering at these high eKE can indeed be deduced from the experimental data because the PAD for the liquid phase remains different from that of the gas phase up to an eKE of $300 \mathrm{eV} . \underline{26}$ We will return to this point below.

It is also interesting to note that our EMFP and IMFP values display a very similar energy dependence as the literature values, but are consistently higher by a factor of $\sim 2-3$. This means that both sets of data, although completely independent, agree on the ratio of IMFP to EMFP, hence on $\left\langle N_{\text {ela }}\right\rangle$. Assuming that this ratio is correct, the vertical offset of our values of EMFP and IMFP can be traced back to the experimental EAL since, as we showed above, both the EMFP and the IMFP scale linearly with the input EAL for a fixed $\left\langle N_{\text {ela }}\right\rangle$. A reduction of the EAL by a factor of $\sim 3$ would bring our EMFP and IMFP values into agreement with the previous estimates. What speaks in favor of such a correction are the expectations that (i) the dielectric formalism used to determine the IMFP from the measured energyloss function of liquid water should be accurate above $100 \mathrm{eV}^{\sqrt{22}}$ and (ii) the EMFP of liquid water might be expected to converge to that of isolated molecules for sufficiently high kinetic energies of a few hundred $\mathrm{eV}$, where the electron scattering is dominated by core-shell electrons that are not affected by solvation and multiple scattering is negligible. What speaks against such a correction are the experimental results of Michaud et al. $\stackrel{29}{ }$ on amorphous ice, which would then lie even much further away from those for liquid water.

The bottom panel of Figure 2 shows the average number of elastic collisions $\left\langle N_{\text {ela }}\right\rangle$ obtained from the present results (triangles) in comparison to a previous analysis of the measured $\beta$ parameters for gas-phase and for liquid water

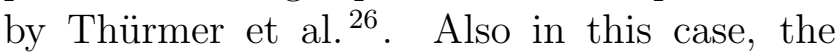
$\left\langle N_{\text {ela }}\right\rangle$ agree reasonably well. This is, however, a coincidence. In the analysis of the experimental results by Thürmer et al. ${ }^{26}\left\langle N_{\text {ela }}\right\rangle$ 
was estimated by successive convolution of the PAD of the gas phase with a DCS originating from an educated guess until the measured PAD of the liquid was obtained. This analysis has two potential deficiencies: A minor problem is that a convolution with the DCS to account for multiple collisions is incorrect for the threedimensional problem. Instead, for a DCS $D_{\mathrm{S}}(\theta)$ with polar angle $\theta$, the angular distribution in the bulk $D^{(n+1)}(\theta)$ after $n+1$ collisions is obtained from that after $n$ collisions as

$$
D^{(n+1)}(\theta)=\int_{0}^{\pi} \int_{0}^{2 \pi} D^{(n)}(\vartheta) D_{\mathrm{S}}\left(\vartheta^{\prime}\right) \sin (\vartheta) d \vartheta d \varphi
$$

with

$$
\cos \vartheta^{\prime}=\cos (\vartheta) \cos (\theta)+\cos (\varphi) \sin (\vartheta) \sin (\theta) .
$$

The difference to a convolution is small but noticeable, as shown in the supplemental material.

More important is the neglect of surface effects in the analysis of Thürmer et al. ${ }^{26}$, as (5) is only valid in the bulk. Due to of the relatively short EAL, in the experiment the contribution from water molecules ionized close to the surface is important. If initially emitted in the direction of the detector, the corresponding electrons cannot scatter as often as those ionized deeper inside the bulk. Hence, to generate the same final angular distribution electrons ionized deeper in the liquid have to contribute, which requires more elastic collisions and, consequently, a larger IMFP.

The influence of the surface is illustrated in Fig. 3, where the PAD is shown after $n$ collisions as determined inside the liquid and outside the liquid, for an eKE of $20 \mathrm{eV}$ and for an EMFP of $0.2 \mathrm{~nm}$. For the latter case, an average over the distance of the ionization site from the surface was performed. While in the bulk about four collisions are needed to obtain the measured distribution of the liquid, more than 10 collisions are needed if the surface effect is taken into account. The inclusion of the surface in the simulation is thus crucial for the analysis of the measurement. Nevertheless, the differ- ence of the $\left\langle N_{\text {ela }}\right\rangle$ shown in Figure 2 is small, because we find larger EMFP values compared to the values used by Thürmer et al. 26 . Before concluding, we note that the EMFP and IMFP values determined in our work are related to physical ab-initio DCS used in our simulations. This must be kept in mind, both when comparing our results with other sources, some of which assume isotropic scattering and the associated transport cross sections, $\stackrel{45}{ }$ and when using our results in simulations.

In conclusion, we have introduced a novel, straightforward, and general method to obtain the EMFP and IMFP of electrons scattering in liquids that is based on the simulation of two types of experiments. Our approach is possible due to the discovery of a rapid convergence of the DCS of water clusters with cluster size, which provides the first ab-initio molecularlevel description of electron scattering in liquid water. It is found that to be consistent with the measured EAL and PAD of soft-Xray photoelectron experiments, the liquid-phase IMFP must be larger than the latest estimate from the dielectric formalism ${ }^{22}$ and the liquidphase EMFP must be larger than that obtained from scattering data of gas-phase water, 11 respectively. We also find that on average only few elastic collisions are possible before inelastic scattering occurs even for relatively low eKE, but that elastic scattering remains important for eKE values up to $300 \mathrm{eV}$. In the studied photoelectron experiments, the importance of elastic scattering is accentuated by the surface sensitivity resulting from the EAL.

The reliability of the mean free paths obtained in our work depends on the accuracy of the experimental input as well as on the accuracy of the DCS used for bulk liquid water. The sensitivity of the retrieved mean free paths on the input parameters is illustrated in Figure S3 of the supplemental material. Hence, further experimental studies would be highly desirable and we hope that our analysis will stimulate additional measurements, also on other liquids. The program code used for this work is available as Python module at https://gitlab. com/axelschild/CLstunfti and can be used to obtain improved values for the EMFP and 

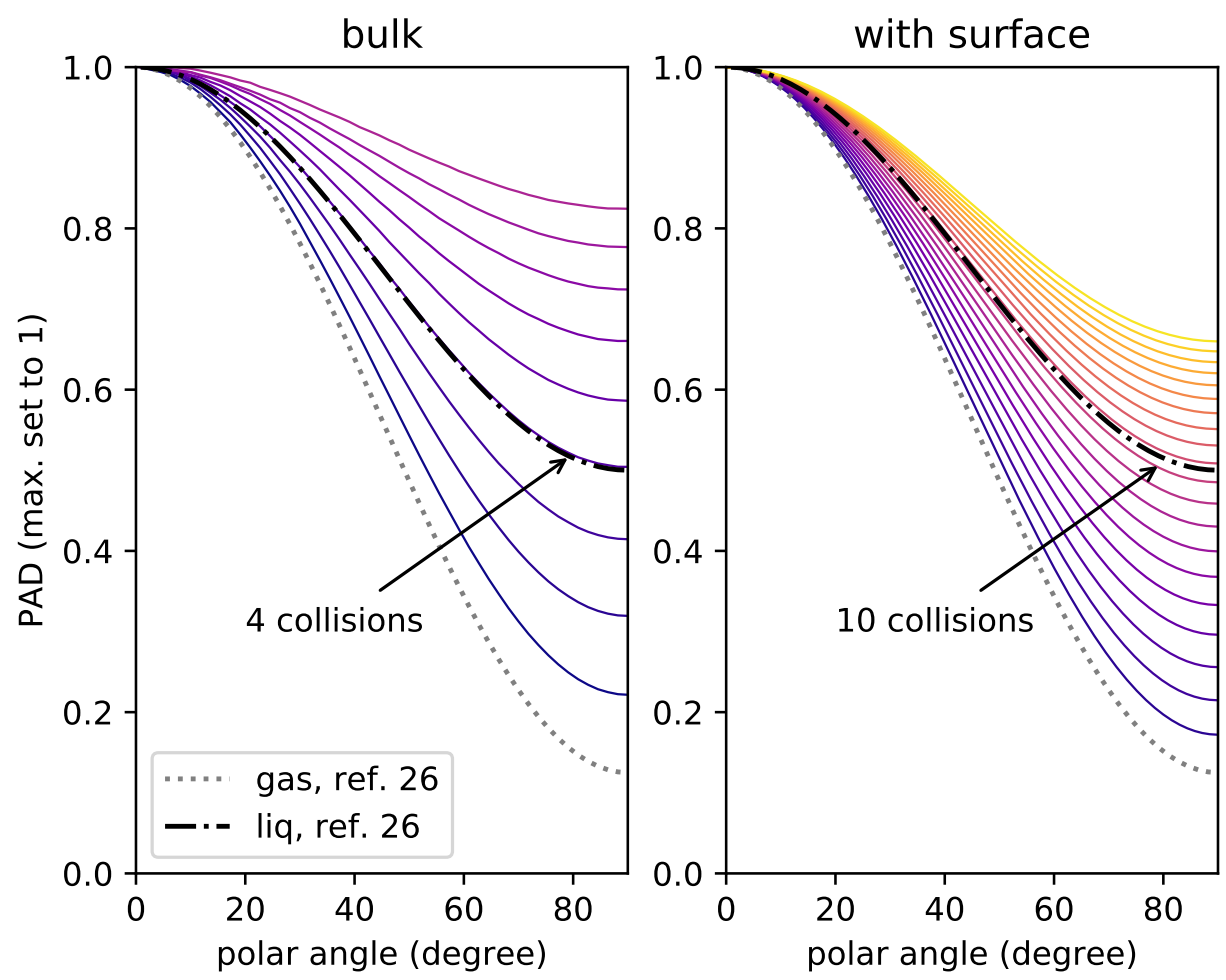

Figure 3: Photoelectron angular distribution (PAD, scaled such that the maximum is 1) for liquid water after scattering in the bulk only (left) and determined outside the liquid (right). The kinetic energy of the scattered electron is $20 \mathrm{eV}$ and the elastic mean free path is set to $0.2 \mathrm{~nm}$. Starting from the initial PAD (dotted line) each line above corresponds to one additional collision. The dotted and dash-dotted line correspond to measured PADs of the gas-phase and the liquid-phase oxygen-1s-electrons from Thürmer et al. 26 . 
IMFP when additional experimental or theoretical data becomes available. We expect that our method to determine the mean free paths will become a powerful tool for understanding electron scattering in liquid media, when combined with accurate experimental and theoretical input data. This opens the door for gaining better knowledge of the properties of liquid water, for the exploration of the scattering properties of other liquids, and for the interpretation of attosecond time-resolved measurements in the liquid phase. $46 \mid 47$

\section{Acknowledgement}

AS thanks Jakub Kocák (ETH Zürich) for helpful discussions, Denis Jelovina (ETH Zürich) for providing some structures of the considered water clusters, and the Swiss National Science Foundation for supporting this research with an Ambizione grant.

\section{References}

(1) Wang, C.-R.; Nguyen, J.; Lu, Q.-B. Bond Breaks of Nucleotides by Dissociative Electron Transfer of Nonequilibrium Prehydrated Electrons: A New Molecular Mechanism for Reductive DNA Damage. Journal of the American Chemical Society 2009, 131, 11320-11322, PMID: 19634911.

(2) Kumar, A.; Sevilla, M. D. Proton-Coupled Electron Transfer in DNA on Formation of Radiation-Produced Ion Radicals. Chemical Reviews 2010, 110, 7002-7023, PMID: 20443634.

(3) Nikjoo, H.; Emfietzoglou, D.; Liamsuwan, T.; Taleei, R.; Liljequist, D.; Uehara, S. Radiation track, DNA damage and response - a review. Reports on Progress in Physics 2016, 79, 116601.

(4) Champion, C. Theoretical cross sections for electron collisions in water: structure of electron tracks. Physics in Medicine and Biology 2003, 48, 2147-2168.

(5) Shin, W.-G.; Bordage, M.-C.; Emfietzoglou, D.; Kyriakou, I.; Sakata, D.; Min, C. H.; Lee, S. B.; Guatelli, S.; Incerti, S. Development of a new Geant4DNA electron elastic scattering model for liquid-phase water using the ELSEPA code. Journal of Applied Physics 2018, 124, 224901.

(6) Katase, A.; Ishibashi, K.; Matsumoto, Y.; Sakae, T.; Maezono, S.; Murakami, E.; Watanabe, K.; Maki, H. Elastic scattering of electrons by water molecules over the range 100-1000 eV. Journal of Physics B: Atomic and Molecular Physics 1986, 19, 2715-2734.

(7) Cho, H.; Park, Y. S.; Tanaka, H.; Buckman, S. J. Measurements of elastic electron scattering by water vapour extended to backward angles. Journal of Physics B: Atomic, Molecular and Optical Physics 2004, 37, 625-634.

(8) Khakoo, M. A.; Silva, H.; Muse, J.; Lopes, M. C. A.; Winstead, C.; McKoy, V. Electron scattering from $\mathrm{H}_{2} \mathrm{O}$ : Elastic scattering. Phys. Rev. A 2008, 78, 052710.

(9) Khakoo, M. A.; Silva, H.; Muse, J.; Lopes, M. C. A.; Winstead, C.; McKoy, V. Erratum: Electron scattering from $\mathrm{H}_{2} \mathrm{O}$ : Elastic scattering [Phys. Rev. A 78, 052710 (2008)]. Phys. Rev. A 2013, 87, 049902 .

(10) Ashley, J. Interaction of low-energy electrons with condensed matter: stopping powers and inelastic mean free paths from optical data. Journal of Electron Spectroscopy and Related Phenomena 1988, 46, $199-214$.

(11) Tomita, H.; Kai, M.; Kusama, T.; Ito, A. Monte Carlo simulation of physicochemical processes of liquid water radiolysis. Radiation and Environmental Biophysics 1997, 36, 105-116. 
(12) Dingfelder, M.; Hantke, D.; Inokuti, M.; Paretzke, H. G. Electron inelasticscattering cross sections in liquid water. Radiation Physics and Chemistry 1998, 53, $1-18$.

(13) Akkerman, A.; Akkerman, E. Characteristics of electron inelastic interactions in organic compounds and water over the energy range $20-10000 \mathrm{eV}$. Journal of Applied Physics 1999, 86, 5809-5816.

(14) Pimblott, S. M.; Siebbeles, L. D. Energy loss by non-relativistic electrons and positrons in liquid water. Nuclear Instruments and Methods in Physics Research Section B: Beam Interactions with Materials and Atoms 2002, 194, 237 - 250.

(15) Emfietzoglou, D.; Nikjoo, H. The Effect of Model Approximations on Single-Collision Distributions of Low-Energy Electrons in Liquid Water. Radiation Research 2005, $163,98$.

(16) Emfietzoglou, D.; Nikjoo, H. Accurate Electron Inelastic Cross Sections and Stopping Powers for Liquid Water over the 0.1-10 keV Range Based on an Improved Dielectric Description of the Bethe Surface. Radiation Research 2007, 167, 110.

(17) Emfietzoglou, D.; Kyriakou, I.; Abril, I.; Garcia-Molina, R.; Nikjoo, H. Inelastic scattering of low-energy electrons in liquid water computed from optical-data models of the Bethe surface. International Journal of Radiation Biology 2012, 88, 22-28.

(18) Emfietzoglou, D.; Kyriakou, I.; GarciaMolina, R.; Abril, I. Inelastic mean free path of low-energy electrons in condensed media: beyond the standard models. Surface and Interface Analysis 2015, 49, 410.

(19) Shinotsuka, H.; Da, B.; Tanuma, S.; Yoshikawa, H.; Powell, C. J.; Penn, D. R. Calculations of electron inelastic mean free paths. XI. Data for liquid water for energies from $50 \mathrm{eV}$ to $30 \mathrm{keV}$. Surface and Interface Analysis 2017, 49, 238-252.
(20) Garcia-Molina, R.; Abril, I.; Kyriakou, I.; Emfietzoglou, D. Inelastic scattering and energy loss of swift electron beams in biologically relevant materials. Surface and Interface Analysis 2016, 49, 11-17.

(21) Emfietzoglou, D.; Papamichael, G.; Nikjoo, H. Monte Carlo Electron Track Structure Calculations in Liquid Water Using a New Model Dielectric Response Function. Radiation Research 2017, 188, $188-188-14$.

(22) Nguyen-Truong, H. T. Low-energy electron inelastic mean free paths for liquid water. Journal of Physics: Condensed Matter 2018, 30, 155101.

(23) Ottosson, N.; Faubel, M.; Bradforth, S. E.; Jungwirth, P.; Winter, B. Photoelectron spectroscopy of liquid water and aqueous solution: Electron effective attenuation lengths and emission-angle anisotropy. Journal of Electron Spectroscopy and Related Phenomena 2010, 177, 60 - 70, Water and Hydrogen Bonds.

(24) Buchner, F.; Schultz, T.; Lübcke, A. Solvated electrons at the water-air interface: surface versus bulk signal in low kinetic energy photoelectron spectroscopy. Phys. Chem. Chem. Phys. 2012, 14, 5837-5842.

(25) Suzuki, Y.-I.; Nishizawa, K.; Kurahashi, N.; Suzuki, T. Effective attenuation length of an electron in liquid water between 10 and $600 \mathrm{eV}$. Phys. Rev. E 2014, 90, 010302 .

(26) Thürmer, S.; Seidel, R.; Faubel, M.; Eberhardt, W.; Hemminger, J. C.; Bradforth, S. E.; Winter, B. Photoelectron Angular Distributions from Liquid Water: Effects of Electron Scattering. Phys. Rev. Lett. 2013, 111, 173005.

(27) Signorell, R.; Goldmann, M.; Yoder, B. L.; Bodi, A.; Chasovskikh, E.; Lang, L.; Luckhaus, D. Nanofocusing, shadowing, and 
electron mean free path in the photoemission from aerosol droplets. Chemical Physics Letters 2016, 658, 1 - 6.

(28) Luckhaus, D.; Yamamoto, Y.; Suzuki, T.; Signorell, R. Genuine binding energy of the hydrated electron. Science Advances 2017, 3, e1603224.

(29) Michaud, M.; Wen, A.; Sanche, L. Cross Sections for Low-Energy (1-100 eV) Electron Elastic and Inelastic Scattering in Amorphous Ice. Radiation Research 2003, 159, 3-22.

(30) Gartmann, T. E.; Hartweg, S.; Ban, L.; Chasovskikh, E.; Yoder, B. L.; Signorell, R. Electron scattering in large water clusters from photoelectron imaging with high harmonic radiation. Phys. Chem. Chem. Phys. 2018, 20, 1636416371.

(31) Bartels, D. M. Is the Hydrated Electron Vertical Detachment Genuinely Bimodal? The Journal of Physical Chemistry Letters 2019, 10, 4910-4913.

(32) Liljequist, D. A study of errors in trajectory simulation with relevance for $0.2-$ $50 \mathrm{eV}$ electrons in liquid water. Radiation Physics and Chemistry 2008, 77, $835-$ 853.

(33) Coe, J. V.; Earhart, A. D.; Cohen, M. H.; Hoffman, G. J.; Sarkas, H. W.; Bowen, K. H. Using cluster studies to approach the electronic structure of bulk water: Reassessing the vacuum level, conduction band edge, and band gap of water. The Journal of Chemical Physics 1997, 10\%, 6023-6031.

(34) Chen, W.; Ambrosio, F.; Miceli, G.; Pasquarello, A. Ab initio Electronic Structure of Liquid Water. Phys. Rev. Lett. 2016, 117, 186401.

(35) Gaiduk, A. P.; Pham, T. A.; Govoni, M.; Paesani, F.; Galli, G. Electron affinity of liquid water. Nature Communications 2018, 9 .
(36) Reid, K. L. Photoelectron Angular Distributions. Annu. Rev. Phys. Chem. 2003, $54,397$.

(37) Gianturco, F. A.; Lucchese, R. R.; Sanna, N. Calculation of lowenergy elastic cross sections for electron- $\mathrm{CF}_{4}$ scattering. The Journal of Chemical Physics 1994, 100, 6464-6471.

(38) Natalense, A. P. P.; Lucchese, R. R. Cross section and asymmetry parameter calculation for sulfur 1s photoionization of $\mathrm{SF}_{6}$. The Journal of Chemical Physics 1999, $111,5344-5348$.

(39) Temelso, B.; Archer, K. A.; Shields, G. C. Benchmark Structures and Binding Energies of Small Water Clusters with Anharmonicity Corrections. The Journal of Physical Chemistry A 2011, 115, 1203412046, PMID: 21910428.

(40) Wernet, P.; Nordlund, D.; Bergmann, U.; Cavalleri, M.; Odelius, M.; Ogasawara, H.; Näslund, L. A.; Hirsch, T. K.; Ojamäe, L.; Glatzel, P. et al. The Structure of the First Coordination Shell in Liquid Water. Science 2004, 304, 995-999.

(41) Frisch, M. J.; Trucks, G. W.; Schlegel, H. B.; Scuseria, G. E.; Robb, M. A.; Cheeseman, J. R.; Scalmani, G.; Barone, V.; Mennucci, B.; Petersson, G. A. et al. Gaussian 09 Revision D.01. 2013; Gaussian Inc. Wallingford CT.

(42) Blanco, F.; Muñoz, A.; Almeida, D.; da Silva, F. F.; Limão-Vieira, P.; García, G. Clustering and condensation effects in the electron scattering cross sections from water molecules. International Journal of Mass Spectrometry 2014, 365366, $287-294$.

(43) Sethna, J. P. Statistical Mechanics: Entropy, Order Parameters, and Complexity; Oxford Univeristy Press: Oxford, Great Britain, 2006. 
(44) Märk, T. D.; Hatano, Y.; Linder, F. Atomic and molecular data for radiotherapy and radiation research; IAEA: Vienna, 1995.

(45) Jablonski, A.; Powell, C. Relationships between electron inelastic mean free paths, effective attenuation lengths, and mean escape depths. Journal of Electron Spectroscopy and Related Phenomena 1999, 100, $137-160$.

(46) Jordan, I.; Huppert, M.; Brown, M. A.; van Bokhoven, J. A.; Wörner, H. J. Photoelectron spectrometer for attosecond spectroscopy of liquids and gases. Review of Scientific Instruments 2015, 86, 123905.

(47) Jordan, I.; Jain, A.; Gaumnitz, T.; Ma, J.; Wörner, H. J. Photoelectron spectrometer for liquid and gas-phase attosecond spectroscopy with field-free and magnetic bottle operation modes. Review of Scientific Instruments 2018, 89, 053103. 